\title{
4th generation dental implant research
}

\author{
Joo Young LEE ${ }^{1}$, Seong Hoon KIM ${ }^{1}$, Jong Oh KANG ${ }^{1}$, Seung Min PARK ${ }^{2,3}$, Ju Hyun $\mathrm{KIM}^{3}$, Mi Rae KIM ${ }^{3}$, \\ Kyung Sook HWANG ${ }^{2}$, Yong Duk PARK ${ }^{1,3, *}$ \\ ${ }^{1}$ Dept. of Prev. Dent. School of Dent. Kyung Hee Univ., ${ }^{2}$ Dept. of Dent. Eng. Shinhan Univ., ${ }^{3}$ Uniance's Central Research \\ Institute
}

INTRODUCTION: After Brenemac, an orthopedic surgeon, accidentally discovered a titanium material that was well fused to a rabbit's fracture 60 years ago, implanting an artificial tooth made of titanium in a dental patient was the starting point. This is what we call first-generation implants. The properties of titanium are biocompatible, rust-resistant, and firmly fused to the alveolar bone.

After that, the implant fixture can withstand stronger masticatory force within the alveolar bone and can be fused well, and while researching the convenience of the procedure, the method of placing a screw on the surface of the fixture has been standardized. These are called second-generation implants.

On the other hand, as the spacing and thickness of the thread and the length of the blade were diversified, methods for fast and strong fusion to the alveolar bone were found. A method was devised to maximize the area on the outer surface of the implant fixture, which was born after repeated research. The third-generation implant is a method of coating with hydroxyapatite, which is the same component as alveolar bone, and a method of maximizing the contact area by creating numerous microscopic gaps.

Main discourse: However, the 3rd-generation implants used so far are compared to missing teeth that have been treated with nerves due to deep cavities among natural teeth. It is a tooth with significantly reduced sense and taste, and is very weak against fracture or avoidance reaction by external force Although the recovery of the patient's masticatory power has been excellently improved, the current implant's greatest weakness is that it has no taste, a risk avoidance response is impossible due to a lack of general sense, and thus an inflammatory response is impossible. Therefore, the future direction of implant research will be directed toward implant research that can bring an anatomical and histological structure similar to natural teeth to overcome these shortcomings. This is the 4 th generation implant. The goal is to restore taste and stimulation by restoring nerves and blood vessels to the position closest to the implant as similar to natural teeth as possible. In the future, implants that can contain these nerves and blood vessels will be studied, and this will be the fourth-generation implant.

Keywords: 4th generation implant, histological structure, implant coating

Acknowledgement: This study is supported by Uniance's Central Research Institute.

Copyright (C) 2021. Korean Academy of Preventive Dentistry. All rights reserved.

This is an Open Access article distributed under the terms of the Creative Commons Attribution Non-Commercial License (http://creativecommons.org/licenses/ by-nc/4.0) which permits unrestricted non-commercial use, distribution, and reproduction in any medium, provided the original work is properly cited. 